\title{
Review of: "Medium-term safety outcomes in patients undergoing tension free vaginal tape obturator for stress urinary incontinence in Murcia, Spain. Historical cohort"
}

\author{
Jose ROMAN
}

Potential competing interests: The author(s) declared that no potential competing interests exist.

\section{REVIEW}

Medium-term safety outcomes in patients

undergoing tension free vaginal tape obturator

for stress urinary incontinence in Murcia, Spain.

Historical cohort

Revista Colombiana de Obstetricia and Ginecologia Vo 72 No1 Enero-Marzo 2021 (43-52)

This is not a descriptive historical cohort where 2 groups are compared with one group exposed to some event and the second group with no exposure to such event. This is a retrospective Observational Cross- Sectional study, as they assess all women exposed to a particular surgery, in this case the TOT procedure for stress urinary incontinence and a follow up is performed to assess the cure rate and the quality of life.

The methodology is flawed with the poor number of patients at follow up, that markedly reduces the validity of any possible conclusion

The Clavien-Dindo classification has been validated by the European Association of Urologists but it does not address the intraoperative complications which are important to describe when dealing with a particular surgical technique.

\section{ABSTRACT}

The total number of patients in the study (166) is not mentioned at all in the abstract. The mean age of the patients is only mentioned.

With regard to the incidence of complications; What type of complications? Intraoperative or postoperative complications? Overall complications? 


\section{INTRODUCTION}

The aim of the study is providing information regarding results and safety of the TOT procedure, This has been already widely reported in the Literature.

As the author states that Pelvic floor physiotherapy is recommended as the first line of treatment. Did the patients involved in this study undergo physiotherapy before the surgery?

\section{MATERIALS AND METHODS}

According to the authors the patients were operated on between July 2013 and April 2017

The patients were seen as a follow up in the clinic between September 2019 and November 2019

That makes a follow up of between 14 months and 6 years 4 months and not a follow up of up to 4 years as it is reported. Did patients operate on at 5-6 years before were absent from the study?

The authors of the article are Urologists and not Gynaecologists. Is a Urogynecologist in Spain a Urologist who performs gynaecological surgery? What is the definition of experienced Urogynecologist?

What is the SELENE software? Company? This is not explained

Urodynamic test? Why was it not performed routinely? It was done apparently in selected cases only. That means you are selecting pathology before the study is done

The stress urinary incontinence was not confirmed by urodynamics in all patients and cases of bladder overactivity may have been missed.

What type of urodynamics machine brand was used? Who did the urodynamics test?

What sling brand was used? Material of the sling? Company? Was the same product used for all the patients?

Description of the technique?

How was the tape placed under the urethra?

Was a Cystoscopy done routinely or cystoscopy was not done at all?

How was the postoperative catheterization managed?

How was the surgery performed: General Anaesthesia? Spinal Anaesthesia?

Was it a Day case procedure?

What was the peri-operative pain relief provided as a routine?

\section{RESULTS}

In Table 1 it is written 39 patients with DO or BOA, this looks as a mistake as 30 patients are mentioned elsewhere with this problem.

The complications are not fully described. For example, the sling extrusion. When did it happen and how it was resolved or treated?

I assume the bladder perforation did happen during the surgery? 
What is the definition of bladder outlet obstruction and what was the outcome of the patient who presented with this problem?

There is no description of the postoperative pain, duration and treatment.

The results presented as a cure rate are misleading because of too many patients were lost for follow up

For example, the results that are presented showed:

144 patients seen at 12 months: cured 90 patients: 90/144: "62,5\% cure"

88 patients at 24 months: cured 55 patients: 55/88: "59\% cure"

61 patients seen at 36 months: cured 31: 31/61: “50.8\% cure"

14 patients seen at 48 months: cured 7: 7/14: “50\% cure"

We cannot assume the cure rate only from patients who showed up to follow up. Otherwise from an " $x$ " study of 400 patients we may have a single patient showing up after 5 years of surgery saying that she is cured, so in this case we are not going to conclude that the cure rate at 5 years was $100 \%$.

The above results are not real as the real number of patients is 166

Percentages are NOT real as unfortunately many patients were lost for follow up

The Reality is

166 patients:

Cured 90 patients seen at 12 months, is $90 / 166$ or $54.21 \%$ cure

Cured 55 patients seen at 24 months is 55/166 or $33.13 \%$ cure

Cured 31 patients seen at 36 months is $31 / 166$ or $18.67 \%$ cure

Cured 7 patients seen at 4 years is $7 / 166$ or $4.22 \%$ cure

From the above we are only able to prove that the cure rate at 1 year was $54 \%$ (very low if we compare it with the rest of the literature) but we do not know for sure about the total results as many patients were lost for follow up.

For the above reasons the table 4 presented in the reviewed study is also misleading An overall comparison of QL before and after surgery using means is misleading. You may have a few patients after surgery whose score results are very outside the most common ones but they may be enough to alter the final mean. In fact, the overall postoperative score mean reported in table 4 is 3.84 but the standard deviation is greater than the mean and reported at 5.76 suggesting a high spread-out of the final results.

You cannot present an overall comparison between the patients before and after surgery without a reference to the time after surgery. To begin with; the variances of the groups will be different and the 
patients are more concentrated at 1 year follow up.

The comparison of quality of life before and after surgery needs to be done per patient:

For example

Patient 1 has a specific quality of life score before surgery

The same patient 1 has a different quality of life score 24 months after surgery

The difference between the above scores will present the improvement or deterioration in the QL score of life of the patient at 24 months. Then you analize if this is statistically significant or not.

So, at the end you will have the number of patients who had a significant improvement in QL score for a specific time after surgery

Editing (translation from Spanish to English could be better)

(I have not fully reviewed the editing)

For example: in page 50: it is written: "because the remaining $37.3 \%$ that not achieved cure" it should have been: "that did not achieve cure".

Page 47: It says "mean and standard deviation (SD) or median and rank". I think a better term could have been "range".

Jose ROMAN, MD.

Hamilton,

NEW ZEALAND 